\title{
Desarrollo de competencias sociolingüísticas e interculturales en ELE: propuesta didáctica con blended learning
}

Por: Esp. Lic. Maribel Carmona Herrera', Universidad Nacional Autónoma de México, México; Mtra. Verónica Cruz Rosales², Universidad Nacional Autónoma de México, México y Mtra. Lydia García Martínez ${ }^{3}$, Universidad Nacional Autónoma de México, México

Recibido: $\quad 28$ de abril, 2017.

Aceptado: $\quad 3$ de abril, 2019.

\section{Resumen:}

El presente artículo da a conocer la aplicación de una secuencia didáctica, cuyo objetivo es el desarrollo de las competencias sociolingüísticas e interculturales en el aprendizaje de Español como Lengua Extranjera. La propuesta se aplicó en dos grupos que participaron en los cursos de español del Centro de Enseñanza para Extranjeros (CEPE) de la Universidad Nacional Autónoma de México (UNAM). Se decidió diseñarla en la modalidad blended learning con la finalidad de combinar las actividades de clase con otras que el estudiante puede realizar de manera autónoma fuera del aula. La secuencia didáctica está conformada por unidades temáticas para trabajar aspectos culturales y gramaticales, los cuales están seleccionados de acuerdo con el programa de curso del nivel intermedio de este centro educativo.

Los resultados obtenidos muestran las ventajas de esta propuesta en tres aspectos: el primero fue constatar el interés y la motivación de los estudiantes en profundizar en los aspectos sociales y culturales de la lengua meta. El segundo, la importancia de los cortometrajes como material didáctico, pues permiten acercar al estudiante a realidades de interacción y contextos comunicativos con los que él puede enfrentarse durante su estancia en un país hispano. Por último, los beneficios de combinar la clase presencial con el trabajo autónomo de los estudiantes, el cual se lleva a cabo en la plataforma y los materiales y actividades de aprendizaje diseñados para este propósito.

1 Maribel Carmona Herrera es Licenciada en Informática, Multimedia y Sistemas por la Universidad Nacional Autónoma de México. Es Especialista en Desarrollo de Aplicaciones Multimedia Interactivas por la Facultad de Ciencias de la misma casa de estudios. Labora en el Departamento de Tecnología y Cómputo del Centro de Enseñanza para Extranjeros de la UNAM. Contacto: maribel@cepe.unam.mx.

2 Verónica Cruz Rosales es Licenciada y Maestra en Pedagogía, graduada de la Universidad Nacional Autónoma de México. Actualmente cursa el doctorado en Pedagogía en la misma casa de estudios y labora en el Centro de Enseñanza para Extranjeros, en el departamento de Español, desde hace trece años. Contacto: verocruz@unam.mx.

3 Lydia García Martínez es licenciada en Lengua y Literatura Hispánica por la Universidad Nacional Autónoma de México y Maestra en Lingüística Aplicada por la Universidad el Estado de Illinois. Actualmente se desempeña como docente de español como lengua extranjera en la Universidad Nacional Autónoma de México. Contacto: lydiagam@unam.mx.
Maribel Carmona Herrera, Verónica Cruz Rosales y Lydia García Martínez. Desarrollo de competencias sociolingüísticas e interculturales en ELE: propuesta didáctica con blended learning. Revista Comunicación. Año 40 , volumen 28 , número 1 , enero-junio, 2019. Instituto Tecnológico de Costa Rica. ISSN: 0379-3974 / e-ISSN1659-3820. 


\title{
Development of socio-linguistic and intercultural competencies in SFL: A didactic proposal using blended learning
}

\begin{abstract}
This article proposes the application of a didactic sequence whose objective is the development of the sociolinguistic and intercultural competencies in learning Spanish as a foreign language. The proposal was applied to two groups that participated in the Spanish courses of the "Centro de Enseñanza para Extranjeros" from Universidad Nacional Autónoma de México (UNAM).

The sequence was designed in the blended learning mode in order to combine class activities with other activities that the student can perform independently outside the classroom. The didactic sequence is made up of thematic units to work on cultural and grammatical aspects, and are selected according to the education center's intermediate level course program.

The results show the advantages of this proposal in three ways: the first was to confirm the interest and motivation of the students in deepening the social and cultural aspects of the target language. The second benefit is the importance of the short films as didactic material, as they allow the student to approach realities of interaction and communicative contexts the student may face during their stay in a Spanish-speaking country. Finally, it also revealed the benefits of combining face-to-face classes with the students' autonomous work, which takes place on the platform and uses learning materials and activities designed for this purpose.
\end{abstract}

\section{ANTECEDENTES}

Si hay algo que caracteriza al aprendizaje de una lengua es su carácter social y cultural, así como el desarrollo de destrezas comunicativas que se deben alcanzar para un uso adecuado según los ámbitos en los que el hablante estará involucrado. El Marco Común Europeo de Referencia para las lenguas (MCER) establece que "la competencia comunicativa comprende varios componentes: el lingüístico, el sociolingüístico y el pragmático. Se asume que cada uno de estos componentes comprende, en concreto, conocimientos, destrezas y habilidades." (Instituto Cervantes, 2002, p. 13).

Según lo anterior, para que el estudiante logre alcanzar una adecuada competencia comunicativa es necesario que domine no solo el sistema lingüístico, sino el marco cultural y social en el que usará la lengua, lo cual necesariamente demanda la interacción con personas nativas. De acuerdo con el contexto de su aprendizaje, es decir, ya sea que aprenda español como lengua extranjera (LE), como segunda lengua (L2) o como lengua de herencia (LH), el estudian- te puede aplicar algunas estrategias de aprendizaje para acercarse al uso real de la lengua que aprende. Así, de manera autónoma, puede buscar la oportunidad para escuchar radio, ver películas o programas de televisión, establecer comunicación por cartas o a través de foros electrónicos, redes sociales, visitar páginas de Internet en la lengua meta, etc.

A pesar de que dichos recursos son válidos para reforzar su aprendizaje, la mayoría de ellos requiere que el estudiante ponga en marcha tanto su conocimiento y competencia lingüística, como sus habilidades y conocimientos relacionados con los aspectos sociolingüísticos, pragmáticos y culturales, lo anterior para lograr la adecuada comprensión de los actos de comunicación implícitos en el mensaje. Sin embargo, no todos los estudiantes cuentan con los conocimientos o la inducción necesaria para lograr la interpretación de los mensajes extralingüísticos que se dan en el acto comunicativo.

En este contexto, el profesor experimentado e interesado en que sus estudiantes desarrollen dicha 
competencia, se ve en la necesidad de buscar y aplicar materiales y propuestas didácticas que fomenten su desarrollo; no obstante, las condiciones institucionales tales como el número de horas del curso, la secuencia didáctica de los libros de texto, evaluaciones y estudio de temas que en su mayoría son de índole gramatical, limitan su estudio y abordaje a los tiempos extra de clase que el profesor pueda dedicar.

A partir de lo anterior, y considerando el perfil de los estudiantes del Centro de Enseñanza para Extranjeros (CEPE) de la Universidad Nacional Autónoma de México (UNAM), campus Ciudad Universitaria y Campus Taxco, quienes se encuentran aprendiendo esta lengua -Español- en un contexto de inmersión (L2), se creó una secuencia didáctica que les ofrece diversas actividades de aprendizaje centradas en los aspectos interculturales de la lengua, con la finalidad de ayudarlos a una mejor integración en el contexto sociocultural en el que se desenvuelven. La secuencia didáctica que se comparte en este artículo pone énfasis en dos aspectos pedagógicos importantes en esta área del saber: el primero, parte de la premisa que el aprendizaje de una lengua se enriquecerá en la medida en que el profesor acerque a su estudiante a los diversos ámbitos y contextos de uso, lo que hará que el alumno elabore procesos cognitivos que lo ayudarán a desarrollar competencias comunicativas específicas; el segundo se enfoca en la importancia de integrar materiales auténticos, como apoyo didáctico a la enseñanza, elemento que no solo ayuda a presentar la estructura gramatical en contexto y el uso de forma natural, sino que además, permite conocer, comprender y analizar la parte social y pragmática de la lengua.

Es importante señalar que, si bien diversos manuales de enseñanza de español como lengua extranjera o segunda lengua integran materiales audiovisuales para su enseñanza, la mayoría de ellos se enfoca en su estructura, en donde si bien se presentan contextos de uso, no necesariamente hacen énfasis en los aspectos sociolingüísticos e interculturales. En este sentido, se considera que la mayoría está hecho para la enseñanza del idioma y no necesariamente muestra su uso auténtico en contextos reales de comunicación. Por tal motivo, la secuencia didáctica que aquí se presenta, da prioridad al uso de mate- riales audiovisuales auténticos, los cuales permiten al alumno comprender el uso y significado real de la lengua desde el punto de vista sociolingüístico e intercultural. Además, dan la oportunidad de reforzar los aspectos morfosintácticos de la lengua.

En específico, el cortometraje, material con base en el cual se desarrolla la propuesta, es creado:

por los nativos para los nativos [...] Esta autenticidad, que acerca al aprendiente a una realidad próxima al uso real del lenguaje de un país hispano, la seguridad de que están ante algo que pueden encontrarse en el país de la lengua objeto y la nueva forma de ver el mundo que se transpira de los documentos, les aporta curiosidad y con ello, el querer saber más acerca de esta realidad, por lo que lo sienten más motivador (Soriano Fernández, 2009, p. 39).

Por otro lado, se consideró integrar el uso de las Tecnologías de la Información y la Comunicación (TIC), herramientas con las que se puede integrar una plataforma electrónica y crear un ambiente de aprendizaje que permita la combinación de las clases presenciales con las no presenciales, lo que constituye una modalidad blended learning. El diseño de actividades de aprendizaje en esta modalidad, toma como base el trabajo independiente que pueden realizar los estudiantes con la consulta de los materiales en línea para, posteriormente, Ilevar al aula dudas específicas que el profesor les ayudará a resolver. En este caso, el docente podrá centrar el tiempo de clase no en la consulta de los cortometrajes, sino en atender las preguntas de los contenidos lingüísticos y extralingüísticos de los materiales vistos en los materiales e incluso, dependiendo del nivel, hacer debates sobre la temática.

Según lo expuesto líneas atrás, este artículo da cuenta de la secuencia didáctica que se diseñó y se aplicó en dos grupos de estudio del CEPE. Es una propuesta que deriva de las reflexiones teóricas que han surgido alrededor de la importancia de crear modelos didácticos y metodológicos de enseñanza-aprendizaje que apoyen al desarrollo de la competencia intercultural de nuestros estudiantes. 


\section{CONTEXTO DE LA ENSEÑANZA}

El Centro de Enseñanza para Extranjeros (CEPE) UNAM, fue fundado en 1921 con la finalidad de "universalizar el conocimiento sobre la lengua española y la cultura mexicana, así como apoyar académicamente a las comunidades mexicanas en el exterior" (Centro de Enseñanza para Extranjeros, 2017, par.1). Así, desde hace 95 años, este Centro se ha dedicado a sistematizar la enseñanza del español como lengua extranjera en un programa de estudio que atiende tanto a los lineamientos establecidos en el MCER, como a la necesidad de integrar contenidos académicos bajo la premisa de promover la cultura mexicana. Así,

la enseñanza del español como lengua extranjera vinculada con las áreas de cultura -Arte, Historia, Ciencias Sociales y Literatura- ha sido una prioridad [...] Dicho objetivo quedó ratificado en la reestructuración del programa de estudios en donde se establece la importancia de mantener una perspectiva interdisciplinaria que integre el aprendizaje de la lengua y la cultura, puesto que el estudiante al haber elegido a nuestro país para aprender el español como segunda lengua, también ha optado por vincularse con el entorno cultural y con aquéllas características que distinguen a México del resto de las naciones hispanohablantes (Centro de Enseñanza para Extranjeros, 2006, p. 3).

Para lograr lo anterior, el programa de estudios del CEPE establece que, entre los objetivos de la enseñanza de la cultura, está el que el estudiante sea capaz de:

- Reconocer las normas sociales que rigen los usos lingüísticos, incluyendo el uso adecuado de diferentes registros.

- Desarrollar un sistema que le permita interpretar adecuadamente los elementos contextuales de la lengua incluyendo intenciones de los interlocutores, tonos afectivos y referencias culturales.

- Desarrollar un conocimiento general adecuado a los contenidos socioculturales básicos de la realidad mexicana e hispanoamericana actual que le permitan comprender la complejidad histórica, literaria y artística de México.

- Aproximarse sin prejuicios a la realidad mexicana (Centro de Enseñanza para Extranjeros, 2006, p. 15).

En la siguiente tabla queda representada la estructura de los cursos del CEPE, en donde se puede observar la carga horaria destinada para los cursos de lengua y cultura:

Tabla 1

Resumen de cursos del programa de estudios del CEPE

\begin{tabular}{|c|c|c|c|c|c|}
\hline Nivel & Curso & Equivalente de MCER & Horas de español & Horas de cultura & Total de horas \\
\hline \multirow{4}{*}{ Básico } & Español 1 & A1 Acceso & 90 & & \multirow{8}{*}{108} \\
\hline & Español 2 & A2 Plataforma & 90 & & \\
\hline & Español 3 & B1 Umbral & 90 & & \\
\hline & Español 4 & & 90 & 18 & \\
\hline \multirow{3}{*}{ Intermedio } & Español 5 & \multirow{3}{*}{ B2 Avanzado } & 78 & \multirow{3}{*}{30} & \\
\hline & Español 6 & & 78 & & \\
\hline & Español 7 & & 78 & & \\
\hline Superior & Español 8 & C1 Dominio operativo / óptimo & 60 & 48 & \\
\hline
\end{tabular}

Información tomada y adaptada del Programa de estudios de los cursos de español y cultura mexicana del Centro de Enseñanza para Extranjeros, UNAM.

Como puede observarse en el cuadro anterior, la mayor carga horaria en todos los niveles está destinada al estudio del sistema lingüístico del español, aunque se dedican algunas horas a la enseñanza de la cultura mexicana. En proporción, es un $17 \%$ de horas de cultura en los niveles básicos, en relación con las 
horas totales de enseñanza de la lengua. Asimismo, se nota que este porcentaje de horas dedicado la enseñanza de la cultura va aumentando conforme el estudiante avanza en los niveles de español, y llega a un $28 \%$, en los niveles intermedios y a un $45 \%$ de horas de enseñanza de la cultura en el nivel superior.

En el programa de estudios del CEPE se menciona también que su enfoque teórico tiene dos grandes ejes. El primero de ellos hace referencia al concepto de competencia, tal como se entiende de manera general en el ámbito educativo (conjunto de conocimientos, habilidades, aptitudes y valores que el estudiante pone en juego para aprender y construir conocimiento). El segundo, de manera más específi$\mathrm{ca}$, trata la competencia comunicativa, la cual tiene relación directa con el aspecto lingüístico. En este contexto, la competencia comunicativa,

comprende el conjunto de conocimientos y capacidades que permite emitir y entender los mensajes de manera contextualmente apropiada; es decir, el conocimiento de las creencias y situación del interlocutor, la negociación de significados, el uso de las fórmulas de tratamiento, el uso de los distintos registros adecuados a la situación y al interlocutor, así como el empleo de fórmulas fijadas para una situación socialmente determinada (Centro de Enseñanza para Extranjeros, 2006, p. 7).

Empero, lograr que el estudiante alcance este nivel de apropiación de la lengua y la cultura requiere de una metodología de enseñanza que integre tanto el sistema lingüístico como los matices pragmáticos, sociolingüísticos y culturales de los contextos en los que se desarrolla la comunicación. De igual modo, es necesario que el estudiante tenga contacto directo con diversos ámbitos de interacción para que se apropie de este conocimiento mediante la experiencia. Este elemento muchas veces no se logra principalmente si el estudiante aprende español como $\mathrm{LE}$, pues el contacto que tenga con el uso real de la lengua puede estar limitado a la institución donde aprende.

Una de las ventajas que se tiene en el contexto de enseñanza en el CEPE es que la mayoría de nuestros estudiantes están en inmersión, de ahí que la posibilidad de que interactúe en diversos ámbitos de comunicación es mayor. No obstante, esta posibilidad dependerá de los motivos de estancia en México de nuestros estudiantes (si realizan estudios universitarios, si están de vacaciones o si su motivo de estancia es por trabajo, etc.), así como de las características culturales que definan a su propio país de origen, pues como sabemos, algunas culturas -como las asiáticas- suelen conservar grupos de interacción cerrados, aun cuando lleven a cabo una vida cotidiana en el país extranjero, lo cual puede deberse a la metodología de la enseñanza de un idioma que llevan a cabo en sus propias culturas. Para ejemplificar lo anterior se ofrece el caso de China:

La enseñanza en la República Popular China se puede sintetizar, hasta ahora, en una palabra: estructuralismo - enseñanzas centradas en la adquisición de la lengua a través del estudio de las estructuras léxicas y gramaticales, haciendo uso de la memoria y carente de contextos verosímiles. Ya sea el aprendizaje de su propia lengua como la metodología que se emplea para cualquier otro tipo de materias, todo confluye en un solo proceso de sistematización y memorización (Bega González, 2015, p. 229).

Es importante hacer notar que los grupos del CEPE Ciudad Universitaria y Taxco están conformados por personas mayores de 18 años y hasta los 40 o 45 años (estos últimos en menor proporción). Los estudiantes llegan de sesenta y nueve distintas nacionalidades, sin embargo, las que predominan son asiáticas (chinos, coreanos, japoneses), que representan un $42.7 \%$ del total de los estudiantes, así como de Estados Unidos y Alemania, quienes aportan un 20\% de la población total (Centro de Enseñanza para Extranjeros, 2017, p. 30).

La propuesta didáctica para el desarrollo de la presente competencia intercultural se dirige a estudiantes de los niveles B1 o B2, según el MCER, ya que estos alumnos empiezan a desarrollar tanto la competencia sociolingüística como la pragmática. La primera abarca el conocimiento y las destrezas para abordar el aspecto social del uso de la lengua, por ejemplo, las normas de cortesía, las diferencias significativas 
que hay entre las costumbres, las actitudes y creencias, entre otros elementos; mientras que la segunda cubre el conocimiento para organizar, estructurar y ordenar un mensaje, utilizar adecuadamente las funciones comunicativas y, por último, crear secuencias de acuerdo con los esquemas de interacción y transacción (Instituto Cervantes, 2002, pp. 119-120). Ambas competencias se pueden desarrollar o evaluar en la expresión e interacción oral y escrita.

Por otro lado, como se puede notar en la Tabla 1, es a partir de los niveles intermedios que se dedican más horas al estudio de la cultura, pues los estudiantes tienen más elementos lingüísticos para participar en debates, compartir opiniones, intereses, hablar de la propia cultura y establecer comparaciones de su cultura con la de la lengua meta. No obstante, la profundidad con la que se trabaje el desarrollo de la competencia intercultural depende de varios factores, entre ellos:

- El tiempo de estancia de los estudiantes, pues algunos de ellos solo asisten a uno o dos niveles y después regresan a su país de origen, de ahí que, aunque pertenezcan a los niveles intermedios, su interés está más enfocado a la adquisición de los aspectos lingüísticos que les permitan consolidar un nivel de comunicación sistemático, con énfasis en los aspectos gramaticales, más que en el desarrollo de la competencia intercultural.

- El interés que tenga el estudiante en el conocimiento de las dinámicas sociales que se generan en los distintos ámbitos de interacción, pues como ya se ha mencionado, aunque su tiempo de permanencia en el país sea largo, sus círculos sociales pueden ser cerrados a los miembros de su misma cultura.

Tomando en consideración estos elementos que circunscriben al contexto de enseñanza y al desarrollo de la competencia intercultural de los estudiantes del CEPE, se pensó en crear una propuesta didáctica que dé la posibilidad de atender a esos estudiantes con un mayor tiempo en México y que están interesados en profundizar en el desarrollo de dicha competencia, sin desatender a quienes estén por un periodo más corto o que sus intereses de aprendizaje sean diferentes. En el siguiente apartado se explica en qué consiste dicha propuesta de enseñanza-aprendizaje.

\section{PROPUESTA DIDÁCTICA CON BLENDED-LEARNING}

Una de las razones que dio origen al diseño de esta propuesta, es el tiempo de permanencia en el país de los estudiantes del CEPE. Se ha detectado que un gran porcentaje de ellos acude a los programas de español de nuestro Centro y toma más de tres cursos, de ahí que su tiempo de permanencia en el país sea de un mínimo de tres meses. Para confirmar lo anterior, se diseñó un cuestionario con el cual fue posible conocer el perfil y los motivos de estancia en el país de los estudiantes, así como las razones por las cuales aprenden español. Dicho instrumento se aplicó a dos grupos de estudio de niveles intermedios (25 estudiantes en total). Una de las preguntas planteadas fue cuánto tiempo tenían de vivir en el país, y los resultados obtenidos señalan que el $31 \%$ de los estudiantes vive en México de 1 a 3 años, y que el $38 \%$ lo hace entre 3 y 6 meses, tal como se detalla en la siguiente gráfica:

\section{Gráfico 1 \\ Tiempo de vivir en México 1}

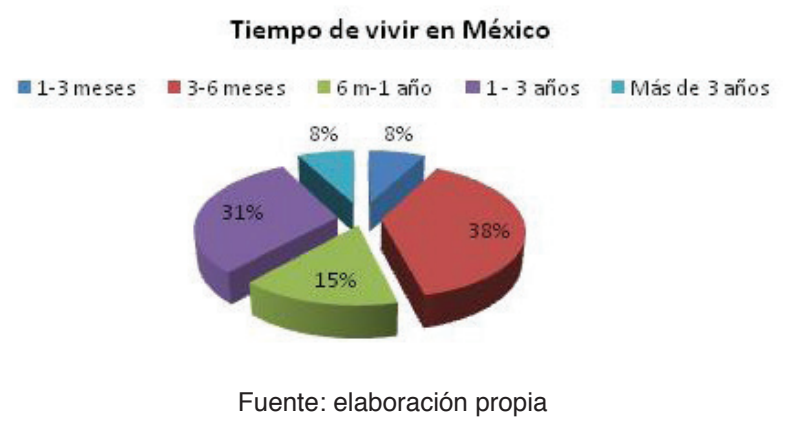

Es importante hacer notar que ante la pregunta planteada a los estudiantes de español, sobre las razones por las cuales residen en este país, el 36\% de los encuestados adujo motivos de trabajo, y otro $36 \%$ indicó "Otras razones", entre las que destaca una relación sentimental con un ciudadano mexicano: 


\section{Gráfico 2}

Motivo para residir en México 1

\section{Razón por la que vive en México}

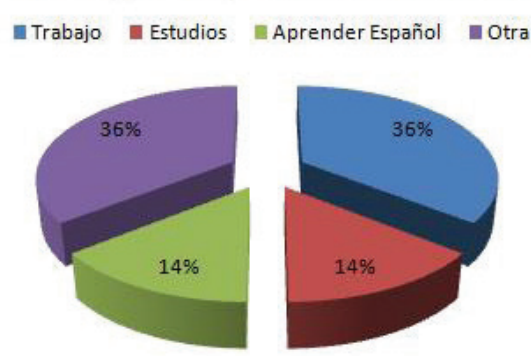

Fuente: elaboración propia

Los anteriores resultados confirman la necesidad que se ha detectado de reforzar en los cursos la competencia intercultural, la cual demanda,

la necesidad de desarrollar en el aprendiente destrezas sociales en los encuentros interculturales. El objetivo es lograr que el aprendiente se comporte de tal manera respecto a las normas y a las convenciones de la comunidad de habla en cuestión que pase por ser un miembro más de ésta. Desde este enfoque la lengua es concebida como un obstáculo para la comunicación entre las personas de culturas diferentes. En cambio, la propuesta del segundo enfoque consiste en desarrollar en el aprendiente ciertos aspectos afectivos y emocionales, entre los que destaca una actitud, una sensibilidad y una empatía especiales hacia las diferencias culturales (Centro Virtual Cervantes, 2017, par. 3).

Esta necesidad parte de la premisa de que los sujetos estudiados, al tener un tiempo prolongado de estancia requieren desarrollar una competencia comunicativa que les permita poder expresar e intercambiar información básica, o ideas sobre temas tanto sencillos como complejos, o acuerdos y desacuerdos; comprender y reaccionar ante actitudes y tonos de los hablantes de la cultura meta.

Por otro lado, y para asegurar de que se tiene el interés de los estudiantes en profundizar en el desarrollo de esta competencia, se les preguntó si durante su estancia habían tenido alguna experiencia intercultural, y si estaban interesados en conocer más sobre

estos temas. A la primera pregunta, el $92 \%$ de los encuestados respondió afirmativamente, tal como se nota en la siguiente gráfica:

\section{Gráfico 3}

¿Ha vivido alguna experiencia intercultural durante su estadía en México?

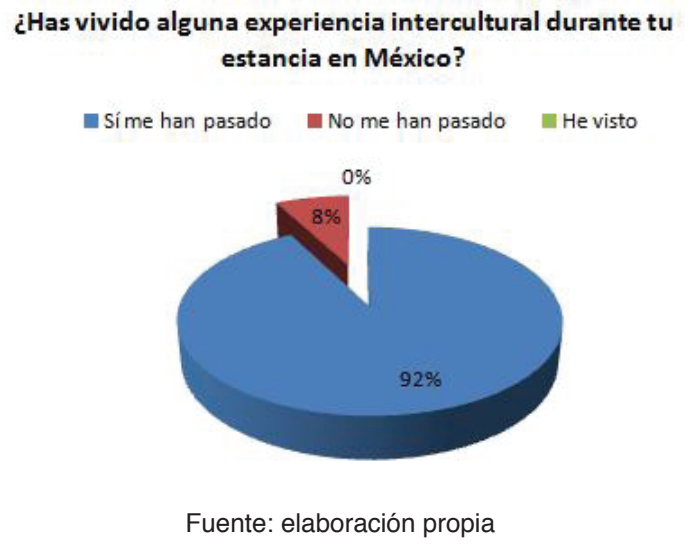

Importante es hacer notar que algunos de los ejemplos dados con respecto a las experiencias vividas, fueron los siguientes:

- Siempre en el restaurante toman propina sin preguntarnos o más que queramos dar.

- Al principio no sabía que tenía que dar propina y todavía me molesta un poco el tema de las propinas.

- A veces tengo confusión sobre cómo presentarme a personas o decir "hola" o adiós, especialmente besarlos a otras personas o no.

- Normalmente no hay cultura de dar propina en Corea, entonces siempre estoy confundida. Creo que los mexicanos no admiten sus culpas.

- La gente se acerca a una persona desconocida de manera rara para mí. Se acercan demasiado.

- Al dar propina, saber cuándo agradecer, en las compras del supermercado, en la escuela, al firmar el contrato de mi depa.

- La gente no dice directamente lo que quiere. A veces se ofenden si uno lo dice directamente. 
Se preguntó también si después de la experiencia vivida preguntaron a alguna persona mexicana sobre lo sucedido. El 46\% respondió que siempre pregunta sobre lo sucedido, y un 31\% que no pudo hacerlo, pero que se muestran interesados en entender la situación, tal como se ve a continuación:

\section{Gráfico 4}

Interés en la situación vivida

\section{¿Por qué preguntaste?}

- Pregunto porque me interesa conocer las costumbres y forma de pensar

- Me sorprendió la situación, pregunté por curiosidad

En mi país no pasan esas cosas y quise entender la situación

- En mi país pasan esas cosas pero la gente actúa diferente

- Otra: sólo pregunto si me parece apropiado

$=5 / \mathrm{r}$

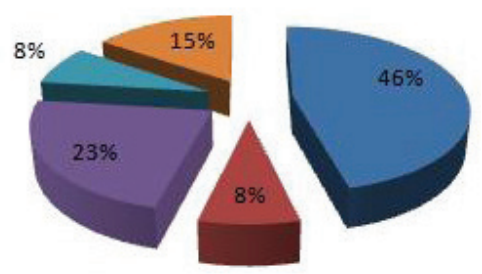

Fuente: elaboración propia

Por lo anterior, y atendiendo al interés manifestado, la propuesta que se diseñó tiene como objetivo ofrecer actividades de aprendizaje centradas en el desarrollo de la competencia intercultural de la lengua, con la finalidad de ayudar a una mejor integración en el contexto sociocultural en el que se desenvuelve. Para lograrlo, se pensó en crear una dinámica de estudio que combine las clases presenciales con actividades fuera de clase, apoyadas por el uso de las tecnologías de información (TIC) y por materiales audiovisuales, pues son idóneos por los vastos recursos que ofrecen en imágenes, gestos, mímica, expresiones faciales, con los cuales es posible interpretar los mensajes y contextualizar la comunicación. La propuesta tecnológica considerada para este propósito se basa en la instrumentación de una plataforma como medio de desarrollo de actividades complementarias para utilizarlas fuera del salón de clases.

Dicha herramienta ofrece la posibilidad de integrar distintas tecnologías, tales como visualizadores, reproductores, herramientas, editores de contenido, formatos, servicios, etc., que permiten la creación de nuevos productos, en este caso educativos. De esta manera, se atiende la necesidad de diversificar las propuestas didácticas que se basen en los nuevos modelos de comunicación digital e interactiva (Valenzuela Miranda, 2013).

Para lograr lo anterior, también se optó por orientar el proyecto como una herramienta de producción propia (con una aplicación web desarrollada con PHP, MySQL y HTML5) la cual puede utilizarse en varios contextos por su facilidad y viabilidad del desarrollo, y que además, tiene un costo bajo y se ciñe a los criterios establecidos por la UNAM. Su diseño permite que los materiales estén abiertos con la finalidad de que los profesores generen materiales de manera independiente, respondiendo a necesidades de diseñar mecanismos y lineamientos que incentiven su uso, con el trabajo coordinado de comunidades académicas que fortalezcan el trabajo colaborativo. Líneas abajo se describe la planeación y puesta en marcha de dicha propuesta.

\section{METODOLOGÍA DE LA PROPUESTA}

Para el diseño de la propuesta, las autoras de este artículo integraron un equipo para distribuir las funciones en la construcción del modelo de la siguiente manera: responsable del diseño tecnológico de la plataforma, así como de su administración y puesta en línea; experto en contenidos, quien diseñó la secuencia de actividades y fue responsable de seleccionar las temáticas y los materiales por integrar; asesor pedagógico, quien junto con el experto en contenidos trabajó en el diseño del número de actividades, definición de estrategias de aprendizaje adecuadas al tipo de contenido (teórico, procedimental, descriptivo), actividades por integrar y procesos cognitivos por atender (contenidos de repaso, introducción, evaluaciones, autoevaluación, etc.), habilidades cognitivas y comunicativas de los objetivos de aprendizaje.

Por su parte, para la secuencia didáctica, se optó por integrar una modelo blended learning, el cual se define como una "forma de aprender que combina o mezcla la enseñanza presencial con la virtual" (García Aretio, 2004. p. 1). Entre las principales características de esta modalidad de estudio se destacan las siguientes: 
- Uso de los recursos de Internet como herramienta y medio para preparar clases, o parte de ellas: temas, subtemas, actividades, evaluaciones, o herramientas de comunicación relacionadas con la clase.

- Distribución de actividades en línea y lecciones presenciales de un $40 \%$ y un $60 \%$, respectivamente.

- Guía para el estudiante de todas las actividades: programa, fechas de entrega, tareas, etc.

- Posibilidad de incluir contenidos completos o parciales, materiales y actividades de aprendizaje, objetos multimedia, videos, etc.

- Integración de audios, textos, páginas web, evaluaciones, apuntes, etc.

- Herramientas de comunicación: blogs, chats, wikis, portafolios electrónicos, etc.

Otras ventajas que aporta esta modalidad es que incentiva el trabajo independiente de los estudiantes, pues no es necesario que el profesor coincida en tiempo y lugar con ellos, sino que los materiales le guían en la elaboración de las actividades. Además, las dudas que se generen en el trabajo en línea pueden ser aclaradas en el espacio de la clase presencial. Por otro lado, la diversidad de materiales que se pueden integrar favorece los diversos estilos de aprendizaje y así, se dan diversidad de estímulos para los distintos tipos de aprendizaje.

Una vez definido el modelo didáctico, se eligió el tipo de materiales que ayudarían a alcanzar el objetivo. Para su selección, se analizaron las posibilidades didácticas que ofrece la herramienta con respecto a los temas culturales de los libros de texto que se estudian en el CEPE, así como su relación con necesidades específicas expresadas por los estudiantes.

Uno de los criterios aplicados en este momento, fue que la plataforma contara con suficiente contenido para potenciar el trabajo de estructuras gramaticales, lexicales y socioculturales relacionadas con el programa del curso. También se puso especial atención en que representara un reto para los alumnos.

Por las razones anteriormente expuestas, se consideró que el material idóneo para integrar a la secuencia didáctica sería el cortometraje, pues su corta dura- ción permite mantener la atención de los estudiantes: "la condensación de la historia en pocos minutos exige expresividad y una concentración de la tensión para mantener expectante al público. Ello contribuye a motivar al alumnado y a centrar su atención en un solo argumento." (Ontoria Peña, 2007, p. 3). Además, puede ser usado como eje temático de una unidad o ser un material de reforzamiento para el estudiante de lengua. De igual modo, como material didáctico tiene las siguientes características:

- Presenta diferentes registros y situaciones.

- Su duración es superior al minuto pero inferior a la media hora.

- Sus historias se constituyen de una introducción, clímax y conclusión, aunque en la mayoría de las veces se presenta un final abierto, el cual podría resultar una oportunidad para crear una actividad que compruebe la comprensión y creatividad del alumno.

- Permite ser usado en todos los niveles (principiante, intermedio y avanzado).

- Facilita el desarrollo de otras actividades que contribuyan al desarrollo de las habilidades auditivas, orales, escritas y lectoras.

\section{PUESTA EN MARCHA DE LA SECUENCIA DIDÁCTICA EN LA PLATAFORMA}

Dentro de la plataforma, el estudiante encontrará una interfaz con un diseño instruccional que lo guiará en el desarrollo de su trabajo. Esta se divide en unidades didácticas para cada nivel, las cuales contienen una estructura de navegación intuitiva y las siguientes secciones básicas:

- Unidad didáctica: describe de manera general en qué consiste la labor por realizar.

- Objetivos: para cada unidad, se muestran los objetivos que el estudiante deberá alcanzar.

- Forma de trabajo: con instrucciones específicas para que el estudiante organice su trabajo, así como algunas recomendaciones sobre estrategias de aprendizaje .

- Índice de contenidos. 
- Evaluación: para cada actividad o ejercicio.

- Material complementario (lecturas, videos, ligas de interés, etc.).
Además, esta plataforma cuenta con herramientas de administración y comunicación que apoyan tanto al alumno como al profesor en su labor, dentro y fuera del salón de clase. Las imágenes siguientes muestran la organización de las secciones arriba descritas:

\section{Imagen 1}

\section{Vista de la página principal de la plataforma de aprendizaje}

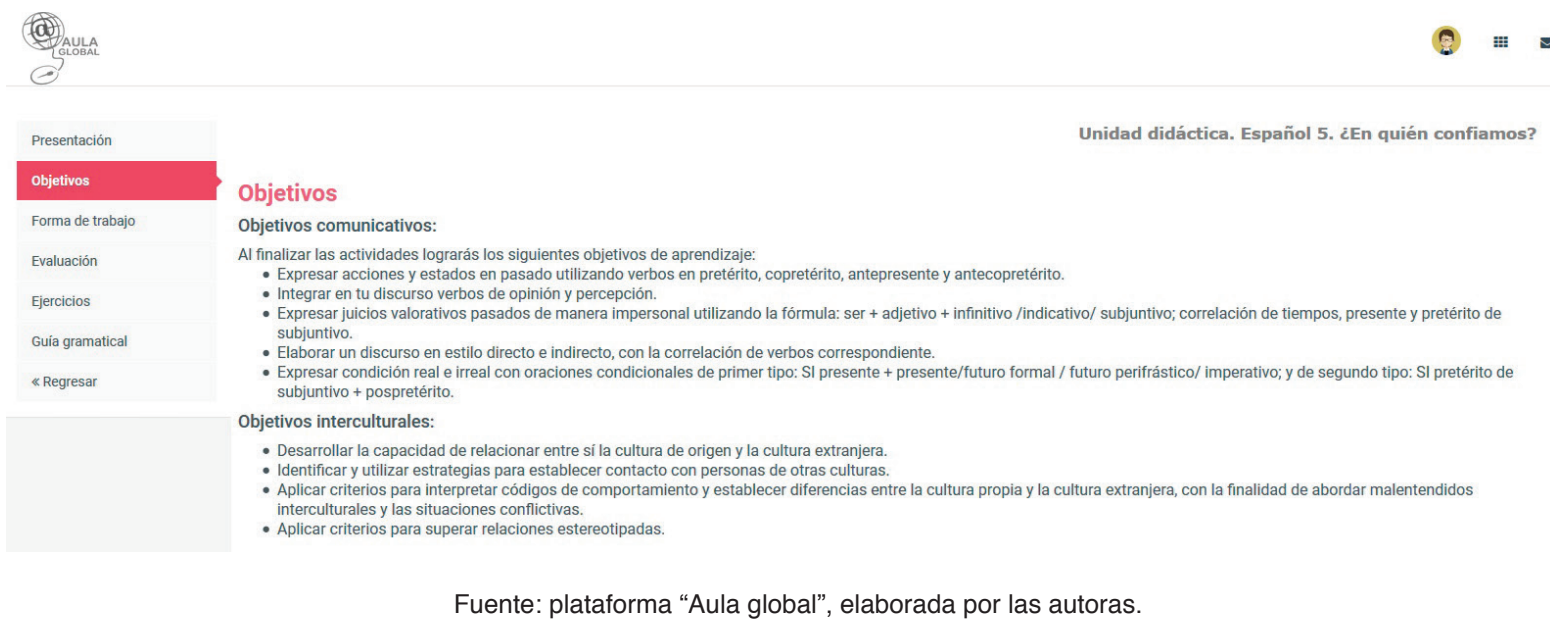

Imagen 2

Pantalla de navegación en las unidades didácticas

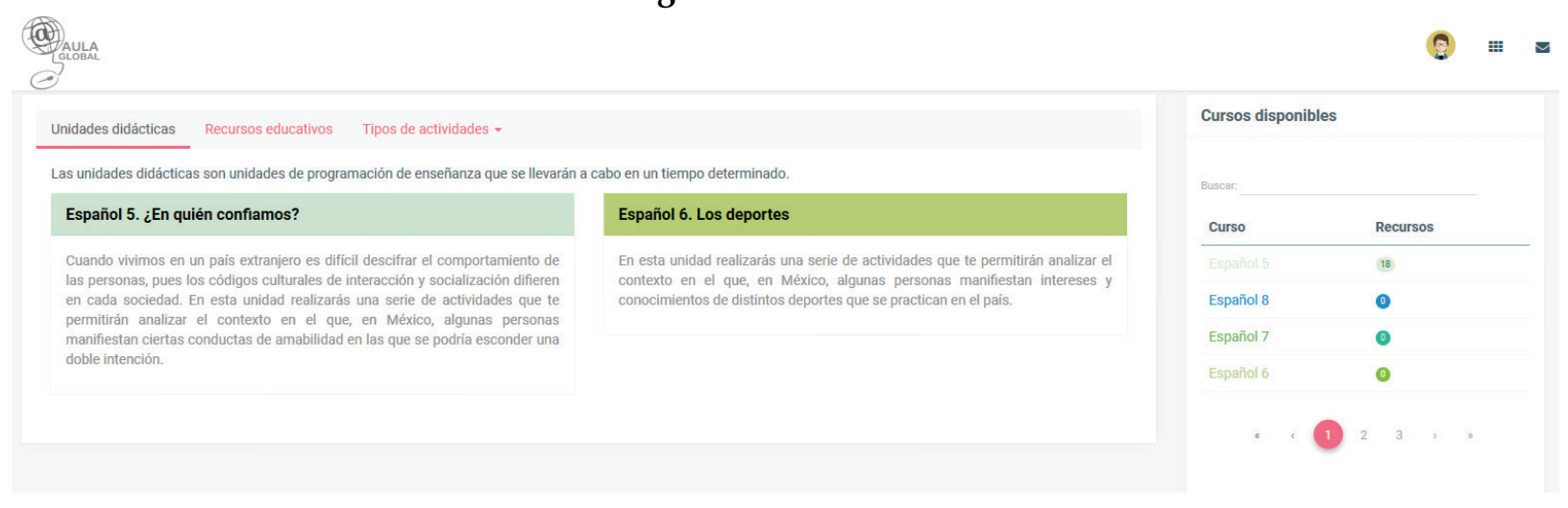

Fuente: plataforma "Aula global", elaborada por las autoras.

Cada unidad didáctica integra una secuencia de ejercicios, tal como se muestra a continuación: 


\section{Imagen 3 \\ Pantalla de navegación de los ejercicios de la unidad}

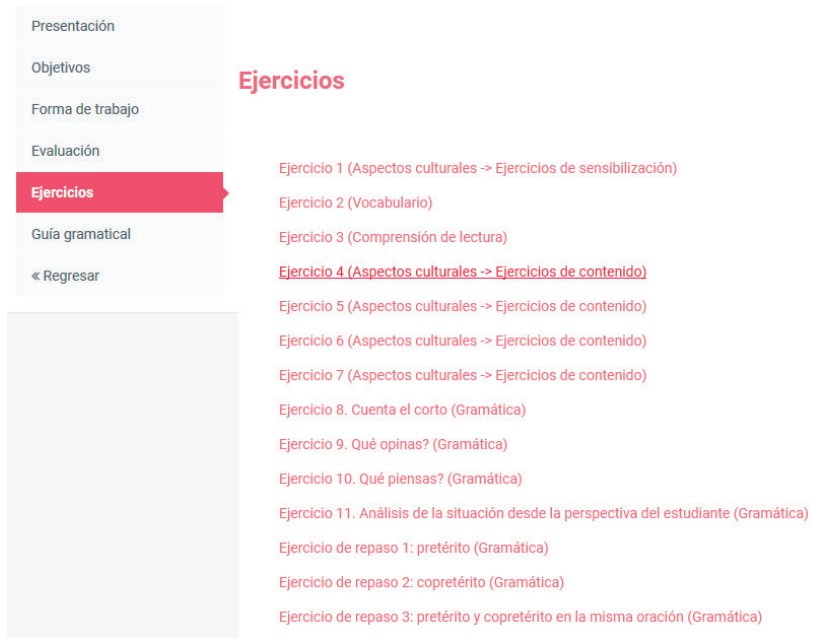

Fuente: plataforma "Aula global", elaborada por las autoras.

Por otro lado, los ejercicios que se integran en la plataforma están diseñados para que el estudiante los elabore fuera de clase y en la plataforma misma. Seguidamente, se describen los tipos que se incluyen:

- Ejercicios de sensibilización al tema: son abiertos, con la finalidad de que los estudiantes hablen lo más que se pueda y presenten situaciones o ejemplos que respalden sus ideas. Su finalidad es que el profesor retome este ejercicio introductorio en clase.

- Ejercicios de vocabulario: Hay de dos tipos: El primero consiste en relacionar columnas para definir la palabra o la frase, y el segundo, permite completar una frase con las opciones presentadas en un cuadro.

- Presentación del cortometraje: los estudiantes pueden verlo cuantas veces lo consideren necesario.

- Ejercicios de contenido sobre el material. En esta parte, hay tres series de preguntas. La primera es de preguntas abiertas, enfocadas a la descripción, situaciones y actitudes de los personajes; la segunda incluye oraciones de respuesta tipo falso o verdadero, sobre particularidades de la historia y con posibilidad de justificar su contestación; la tercera es de situaciones y el estudiante debe elegir la opción que se adecua a su forma de pensamiento. Aquí se observa un primer acercamiento de comparación con la cultura del alumno y la de la lengua meta.

- Presentación de la estructura gramatical: para cumplir con el objetivo comunicativo.

- Ejercicios en contexto para la práctica de la habilidad lingüística presentada. Aquí se incluyen tres series de ejercicios: a) un resumen del cortometraje, donde se escribe la forma correcta del verbo ofrecido, b) un texto que expresa una opinión, y c) una serie de diez oraciones de respuesta libre pero coherente y lógica.

- Señalamiento de los elementos culturales y pragmáticos por comentar. Permite analizar los gestos y los tonos y comentar ampliamente.

- Actividad oral o escrita: aquí se comparan la cultura del estudiante y la de la lengua meta ${ }^{4}$.

Después de que el estudiante elabora de manera individual los ejercicios anteriores, el profesor retoma el tema en clase y plantea algunas actividades que den lugar a la discusión, reflexión, solución de dudas y en sí, a la puesta en práctica de la competencia comunicativa de los estudiantes. Algunas de las actividades que se pueden plantear en el salón de clase son:

- Reflexiones sobre el título del cortometraje, la temática, etc.

- Representaciones de actitudes vistas en el corto, para verificar si se asimiló la parte pragmática y social de la lengua meta.

- Consideraciones sobre los elementos culturales del cortometraje.

- Cavilación sobre los elementos pragmáticos que aparezcan en el material audiovisual.

- Comparación entre la cultura del estudiante y la de la lengua meta.

Para facilitar la lectura de los tipos de ejercicios que se integra-
ron y el nivel cognitivo al que atienden, véase el Anexo: Clasificación de ejercicios en la propuesta didáctica. 
En este punto, es importante hacer notar que aunque los estudiantes estén en inmersión, no significa que existe un constante contacto o interacción con el contexto cultural o con hablantes nativos. Muchos de ellos tienen círculos cerrados de convivencia constituidos por personas de su misma nacionalidad y por eso, el profesor de lenguas viene a ser un representante cultural y debe recurrir a diferentes herramientas didácticas para presentar y explicar las cuestiones culturales de la lengua meta. La propuesta didáctica con blended learning brinda esta posibilidad, pues permite combinar el trabajo independiente de los estudiantes y hacer reflexiones personales, sin descuidar el trabajo en la plataforma.

\section{CONCLUSIONES}

Una vez aplicada esta secuencia didáctica, es posible concluir que la modalidad blended learning permite que el alumno del CEPE-UNAM desarrolle las actividades de la unidad didáctica en dos fases:

1.Trabajo en la plataforma, en el tiempo y ritmo del alumno, para repasar la parte estructural de la lengua. En esta etapa, el estudiante tuvo la oportunidad de ver el cortometraje y resolver los ejercicios de contenido.

2.Trabajo en el salón de clases, en donde el profesor se convirtió en un facilitador de la lengua y la cultura, en este caso, la mexicana. Aquí, se analizaron los aspectos sociolingüísticos y culturales y se comparan las culturas, siempre con el apoyo del profesor. Valga anotar que esta discusión también es una oportunidad para la práctica oral, la cual, al ser moderada por el profesor, facilita la participación de todo el grupo.

Por otro lado, la posibilidad de dedicar un tiempo de la clase presencial para comentar las apreciaciones sobre el cortometraje propuesto, permite que los alumnos contrasten sus opiniones y se enriquezca la perspectiva cultural a partir de la cual analizan las situaciones comunicativas. Asimismo, da lugar para que el alumno ponga en práctica los contenidos lingüísticos que ya domina y prepara para interactuar en contextos reales con hablantes nativos, de modo que es posible desarrollar la habilidad oral sin descuidar la explicación o repaso de la estructura de la lengua. Y, sobre todo, facilita el análisis de los asuntos sociolingüísticos y culturales de la lengua.

Si bien uno de los objetivos que se buscó con la propuesta didáctica aquí presentada es apoyar al estudiante de ELE en inmersión en el desarrollo de sus competencias sociolingüísticas, pragmáticas e interculturales, es también claro que este es un proceso gradual y perfectible, el cual ocurre gracias a la interacción que tenga el estudiante en los diversos ámbitos sociales. Por otro lado, si bien se pueden crear materiales y propuestas didácticas que apoyen al desarrollo de estas competencias, la investigación hecha sobre su evaluación confirma que hacer este seguimiento, junto con la valoración en términos objetivos, no es un proceso sencillo, pues el desarrollo de la competencia lingüística y comunicativa no siempre va de la mano con la competencia intercultural. Es importante considerar que su adquisición demanda una disposición e interés del estudiante por desarrollarla, así como una apertura a la convivencia.

En este sentido, y a partir de la experiencia con la aplicación de esta secuencia, al menos la población de estudiantes que participan de los cursos del CEPE, se encuentra interesada en ampliar su conocimiento de las pautas culturales en diversos contextos de comunicación, y que este ejercicio permitió crear una sesión en donde ellos pudieron expresar sus inquietudes sobre los temas vistos en el material y el profesor puedo tomar nota de otros aspectos interculturales posibles de trabajar.

No es posible afirmar que, después de ver el material, hubo un cambio de opinión entre las ideas o imaginarios que los estudiantes tienen de la cultura mexicana, en particular sobre el tema específico analizado en el cortometraje. Lo que sí se puede concluir es que, con esta modalidad de trabajo y con la posibilidad de llevar al aula el análisis de estos temas, se puede enriquecer la discusión y profundizar en el análisis de diversos elementos culturales que posibilitan, al menos, la reflexión y un conocimiento más amplio de la competencia intercultural y sociolingüística.

De igual modo, se parte de la convicción de que es necesario que el profesor de lengua sensibilice y guíe al estudiante en el conocimiento y apropiación de 
los códigos culturales, no solo por ser un representante de la cultura y la lengua que enseña, sino porque es en el espacio didáctico que se crea en la clase de lengua en donde se pueden analizar y contrastar los referentes de identidad de los grupos sociales en los que el alumno se está integrando. Además, si el grupo es heterogéneo, habrá un conocimiento cultural de cada uno de sus compañeros y una reflexión sobre las coincidencias y diferencias que hay entre el grupo y la lengua meta. La idea es acercar al alumno de manera paulatina a los diversos contextos y ámbitos en los que pueda interactuar con hablantes nativos, y con ello ayudarlo a reforzar estas competencias.

Como ya se ha explicado, la secuencia didáctica considera el uso de los cortometrajes como un material didáctico pertinente, pues tiene como ventaja mostrar ejemplos de comunicación reales y auténticos.

Ante esto, se puede adicionar que al elegir estos materiales hay que considerar que su contenido no incomode a la audiencia con temáticas religiosas, políticas, sexuales y otras sensitivas. Como bien lo señala Pastor (2016):

En las aulas en las que existan diversas culturas, es esencial que se tengan todas en cuenta fomentando un clima de aprendizaje intercultural en el que el alumno sea consciente de sus propias presuposiciones culturales, así como de las de sus compañeros ( $p$. 7).

La propuesta que se ha descrito en este trabajo se encuentra en su primera etapa de aplicación. Por eso, la evaluación para conocer su efectividad se ha pensado en tres momentos:

1)Elaboración de escalas de percepción, para conocer los estereotipos, prejuicios e ideas en general que el estudiante trae sobre México.

2)Interacción con la plataforma tecnológica y las actividades de aprendizaje propuestas en esta herramienta.

3)Contraste de las opiniones previas que tenían los estudiantes con las ideas que han conformado con el trabajo en clase y con su experiencia en el contexto de inmersión. Y aunque los resultados objetivos sobre estas percepciones no será sencillo de obtener (seis semanas de duración de un curso no son suficientes para que el estudiante profundice o interactúe en todos los ámbitos posibles), se cree que el ejercicio dará elementos para evaluar y hacer las mejoras necesarias a la propuesta original.

\section{REFERENCIAS BIBLIOGRÁFICAS}

Bartolomé, A. R. (2004). Blended Learning. Conceptos básicos. Pixel-Bit. Revista de Medios y Educación, 23, 7-20.

Bega González, M. R. (2015). Dificultades metodológicas de los estudiantes sinohablantes de español como lengua extranjera. Onomázein, Revista semestral de lingüística, filología y traducción, 32, 227-232. Recuperado de : http://onomazein.letras. uc.cl/Articulos/N32/32_13_Bega.pdf.

Centro de Enseñanza para Extranjeros CEPE. (2017). CEPE-UNAM. México, CDMX. Recuperado de http://www.cepe.unam.mx/.

Centro de Enseñanza para Extranjeros CEPE. (2017) CEPE-UNAM. México. CDMX. Recuperado de http://www.cepe.unam.mx/archivos/cepe/INFORME_RESULTADOS_2016_17022017.pdf.

Centro de Enseñanza para Extranjeros CEPE. (2006). Programa de estudios de los cursos de español y cultura. Documento no publicado. Consultado en 18 de noviembre, 2017 Recuperado de: http://132.248.130.129/archivos/cepe/ProgramaCEPE.pdf.

Centro Virtual Cervantes. (2017). Diccionario de términos clave de ELE. España. Recuperado de: http:// cvc.cervantes.es/ensenanza/biblioteca_ele/diccio_ ele/diccionario/compintercult.htm.

García Aretio, L. (2004). Blended learning. ¿Enseñanza y aprendizaje integrados? Recuperado de http://e-spacio.uned.es/fez/eserv/bibliuned:333/ editorialoctubre2004.pdf.

Instituto Cervantes. (2002). Marco Común Europeo de Referencia para las Lenguas: Aprendizaje, 
enseñanza y evaluación. Madrid: Ministerio de Educación, Cultura y Deporte.

Ontoria Peña, M. (2007). El uso de cortometrajes en la clase de ELE. RedELE (en línea), nº 9, febrero 2007, Ministerio de Asuntos Exteriores, disponible en: http://www.mecd.gob.es/dctm/redele/MaterialRedEle/Revista/2007_09/2007_redELE_9_06Ontoria. pdf?documentld=0901e72b80df3598.

Pastor Gutiérrez, I. (2016). Actitudes de los alumnos chinos respecto al aprendizaje del español en España. Revista de Enseñanza de español a hablantes de chino. Suplementos. 14. Consultado el 19 de noviembre de 2016 en: http://www.sinoele.org/ images/Revista/14/Suplementos/pastor_TFM.pdf.

Soriano Fernández, S. (2009). Con cortos y sin cortes. Una propuesta didáctica para el uso del cortometraje en la clase ELE (Memoria de máster, Universidad Antonio de Nebrija, España). Recuperada de: https://marcoele.com/descargas/10/cortos_ele_s. soriano.pdf.

Valenzuela Miranda, G. (2013). Análisis y reflexión de la plataforma electrónica y el aprendizaje virtual. Recuperado de: http://www.ri.uson.mx/revistas/articulos/1-1-art5.pdf. 


\section{Anexo: Clasificación de ejercicios en la propuesta didáctica}

\begin{tabular}{|c|c|c|c|c|}
\hline $\begin{array}{l}\text { Nivel cogni- } \\
\text { tivo }\end{array}$ & $\begin{array}{l}\text { Finalidad del } \\
\text { ejercicio }\end{array}$ & $\begin{array}{l}\text { Objetivo de } \\
\text { aprendizaje }\end{array}$ & Forma de trabajo & Tipo de reactivo \\
\hline \multirow[t]{2}{*}{ Identificación } & \multirow{3}{*}{$\begin{array}{l}\text { Sensibilización } \\
\text { al tema }\end{array}$} & $\begin{array}{l}\text { Identificar el tema por tra- } \\
\text { bajar }\end{array}$ & $\begin{array}{l}\text { En línea } \\
\text { No hay respuestas correctas. Se busca } \\
\text { conocer el perfil del estudiante. }\end{array}$ & $\begin{array}{l}\text { Opción múltiple. } \\
\text { Cuadro de texto } \\
\text { para dar opinión }\end{array}$ \\
\hline & & $\begin{array}{l}\text { Identificar vocabulario rela- } \\
\text { cionado con el tema. }\end{array}$ & $\begin{array}{l}\text { En línea } \\
\text { Seleccionar la definición de la palabra. }\end{array}$ & $\begin{array}{l}\text { Relación de colum- } \\
\text { nas. }\end{array}$ \\
\hline \multirow{3}{*}{ Comprensión } & & $\begin{array}{l}\text { Reconocer información re- } \\
\text { lacionada con el tema en un } \\
\text { texto. }\end{array}$ & $\begin{array}{l}\text { En línea } \\
\text { Texto breve relacionado con el tema de } \\
\text { la unidad. Las preguntas relacionadas } \\
\text { son de comprensión. }\end{array}$ & Opción múltiple. \\
\hline & \multirow[t]{2}{*}{$\begin{array}{l}\text { Presentación } \\
\text { del tema }\end{array}$} & $\begin{array}{l}\text { Distinguir e identificar infor- } \\
\text { mación del cortometraje } \\
\text { (el estudiante observará el } \\
\text { cortometraje las veces que } \\
\text { requiera). }\end{array}$ & $\begin{array}{l}\text { En línea } \\
\text { Actividades para identificar informa- } \\
\text { ción general y específica de la historia } \\
\text { vista en el material. }\end{array}$ & $\begin{array}{l}\text { Opción múltiple. } \\
\text { Preguntas F-V. }\end{array}$ \\
\hline & & $\begin{array}{l}\text { Interpretar algunas escenas } \\
\text { del cortometraje (aspectos } \\
\text { interculturales). }\end{array}$ & $\begin{array}{l}\text { En línea } \\
\text { Preguntas sobre tonos de voz, actitudes, } \\
\text { interpretación de frases, gestos. }\end{array}$ & Opción múltiple. \\
\hline \multirow[t]{2}{*}{ Aplicación } & \multirow{2}{*}{$\begin{array}{l}\text { Interpretación } \\
\text { del tema }\end{array}$} & \begin{tabular}{|l|}
$\begin{array}{l}\text { Completar frases integrando } \\
\text { verbos conjugados }\end{array}$ \\
\end{tabular} & $\begin{array}{l}\text { En línea } \\
\text { Textos narrativos de la historia con hue- } \\
\text { cos para integrar verbos conjugados. }\end{array}$ & Cloze. \\
\hline & & $\begin{array}{l}\text { Escoger verbos adecuados } \\
\text { para completar frases de } \\
\text { opinión }\end{array}$ & $\begin{array}{l}\text { En línea } \\
\text { Frases de opinión con huecos para inte- } \\
\text { grar verbos. }\end{array}$ & Cloze. \\
\hline Análisis & \multirow[t]{2}{*}{$\begin{array}{l}\text { Apropiación } \\
\text { del tema }\end{array}$} & $\begin{array}{l}\text { Valorar la información del } \\
\text { material y relacionarla con } \\
\text { la experiencia propia. }\end{array}$ & $\begin{array}{l}\text { En línea } \\
\text { En plataforma hay frases incompletas } \\
\text { para llenar con textos con la opinión de } \\
\text { cada estudiante. } \\
\text { En clase } \\
\text { El profesor revisará el texto libre y dis- } \\
\text { cutirá y contrastará las opiniones de los } \\
\text { estudiantes. }\end{array}$ & Respuesta abierta. \\
\hline Evaluación & & $\begin{array}{l}\text { Contrastar el tema visto en } \\
\text { el material con la cultura } \\
\text { propia del estudiante. }\end{array}$ & $\begin{array}{l}\text { En línea } \\
\text { El estudiante creará un texto con al- } \\
\text { gunas líneas de análisis sugeridas para } \\
\text { comparar el tema visto en el material } \\
\text { con la situación de su país y cultura. } \\
\text { En clase } \\
\text { El profesor retomará las composiciones } \\
\text { de los estudiantes para abrir un debate } \\
\text { sobre las distintas opiniones y experien- } \\
\text { cias. }\end{array}$ & Respuesta abierta. \\
\hline
\end{tabular}

